Original Research Paper

\title{
Piezoelectric Co-Rotational 3-Node Shell Element
}

\author{
Gil Rama, Dragan Marinkovic and Manfred Zehn \\ Department of Structural Analysis, Berlin Institute of Technology, Berlin, Germany
}

Article history

Received: 19-09-2016

Revised: 06-10-2016

Accepted: 08-10-2016

Corresponding Author:

Gil Rama

Department of Structural

Analysis, Berlin Institute of

Technology, Berlin, Germany

Email: gil.rama@tu-berlin.de

\begin{abstract}
Geometrically nonlinear FE-analysis of active piezoelectric thinwalled shell structures, based on a Co-Rotational (CR) formulation and the Cell-Smoothed Discrete-Shear-Gap 3-node shell element (CS-DSG3) is presented in this study. The electric potential across the thickness of piezolayers is approximated by a linear function. The basic assumption of the applied Co-Rotational (CR) FEM formulation is that translations and rotations are finite, whereas the strains are small. Large rotation theory is used to update the rotational degrees of freedom. The proposed formulation is tested by means of the originally developed program. A set of piezoelectric coupled static linear and nonlinear cases are studied and the obtained results are compared with those available in the literature. A very good agreement of the results is noted, whereby the numerical effort could be reduced and numerical stability improved compared to other geometrically nonlinear FEM formulations.
\end{abstract}

Keywords: Finite Element, Piezoelectric, Shell, Co-Rotational, Triangular Shell Element, CS-DSG Element

\section{Introduction}

In engineering practice smart structures are utilized to achieve better operational safety, noise reduction, improved comfort, etc. A number of materials demonstrate sufficiently strong piezoelectric effect, which couples the mechanical to electric field and vice versa, so that they can be employed as multi-functional materials for active elements (actuators/sensors) of smart structures. Particularly piezoelectric ceramics offer the advantages of producing relatively high actuation forces and high efficiency of energy conversion combined with low power consumption. These advantages led to the wide application of piezoelectric ceramics including various types of actuators and sensors for smart structures.

Design of smart structures demands accurate and reliable tools for their modeling and simulation. The Finite Element Method (FEM) has established itself as the method of choice for these problems. Over the past 60 years, the FEM has experienced its evolution whereby its application in the field of structural analysis has been the major driving component of the development. Within the framework of the FEM, various shell elements represent the principle workhorse in modeling thin-walled structures. The main requirements for shell elements are high efficiency, reliability and applicability over a wide range of thickness and curvature. Additionally, thin-walled structures are characterized by high susceptibility to geometrically nonlinear behavior, caused by large transverse deflections and therewith local rotations so that the developed FEM formulations are also supposed to cover this aspect.

Many studies related to piezoelectric structures are based on the linear theories of elasticity and piezoelectricity. Since the pioneer work of Allik and Hughes (1970), various types of finite elements have been developed for modeling piezoelectric smart structures. The survey from Benjeddou (2000) gives a thorough overview of the development in the field during the ' $90 \mathrm{~s}$ and the development proceeded at the same rapid pace in the decades to follow. Just a few of the developments are to be mentioned here in order to illustrate the interest of the researchers in this field. Piefort (2001) has used the layerwise facet element available in the commercial FE package SAMCEF and extended it by including an arbitrary number of piezolayers. The element applies the Mindlin-Reissner kinematics and accounts for the full piezoelectric coupling. Gabbert et al. (2002) have extended a SemiLoof shell element, based on the Kirchhoff kinematics, so that it can be used for modeling piezoelectric composite laminates. Kogl and Bucalem (2003) have proposed a bilinear shell with each node assigned an electrical degrees of freedom. The method of Mixed Interpolation of Tensorial Components (MITC) is used 
to alleviate the locking effects with this element. Mesecke-Rischmann (2004) has proposed a shallow shell element with the electric potential over the thickness of piezoelectric layers considered to be a quadratic function. Marinkovic et al. (2006) have proposed a full biquadratic degenerated shell element for piezoelectric active laminates. This element has been exhaustively used to investigate various aspects of thinwalled smart structures, such as: Adequate approach to describe of the electric field in the piezolayers (Marinkovic et al., 2007), geometrically nonlinear effects (Marinkovic et al., 2008), how mesh distortion affects the results with the piezoelectric coupling involved (Marinkovic and Marinkovic, 2012), etc. The element has also been implemented in the commercially available FEM program ABAQUS (Nestorovic et al., 2014) for the convenience of interested users. Furthermore, a number of researchers (e.g., Rabinovitch, 2005; Kulkarni and Bajoria, 2007) have put focus in their work to geometrically nonlinear effects in mechanical behavior of laminated thin-walled structures with piezolayers.

In this study, the CS-DSG3 Co-Rotational (CR) shell element developed by Rama et al. (2016) is extended to geometrically nonlinear analysis of composite shells integrated with piezoelectric layers. The essence of the applied CR-approach is described by Marinkovic et al. (2012). It offers high numerical efficiency and stability, which is even more pronounced when used in combination with modern hardware solutions for computations (Nutti and Marinkovic, 2014).

\section{Governing Equations for Piezoelectric Composite Plates}

\section{Strain Field}

The position vector $X$ of each point in the shell domain is given by the sum of the position vector of mid-surface $X_{0}^{\text {old }}$ and the transverse direction vector normal to the mid-surface $X_{h}^{\text {old }}$ (Kim and Kim, 2002):

$$
X=X_{0}^{\text {old }}+\xi X_{h}^{\text {old }}
$$

where, the natural coordinate $\xi \in[-1,1]$ indicates the position in the thickness direction. The displacement field $U$ is obtained by the difference of deformed (new) $X_{0, h}^{\text {old }}$ and un-deformed (old) $X_{0, h}^{\text {old }}$ configuration (Fig. 1).

The magnitude of the normal direction vector $X_{h}$ is $t / 2$, with the shell thickness $t$ :

$$
U=\left(X_{0}^{\text {new }}-X_{0}^{\text {old }}\right)+\xi\left(X_{h}^{\text {new }}-X_{h}^{\text {old }}\right)
$$

The vector $U$ is given by the two parts $U_{0}$ and $\xi U_{h}$ :

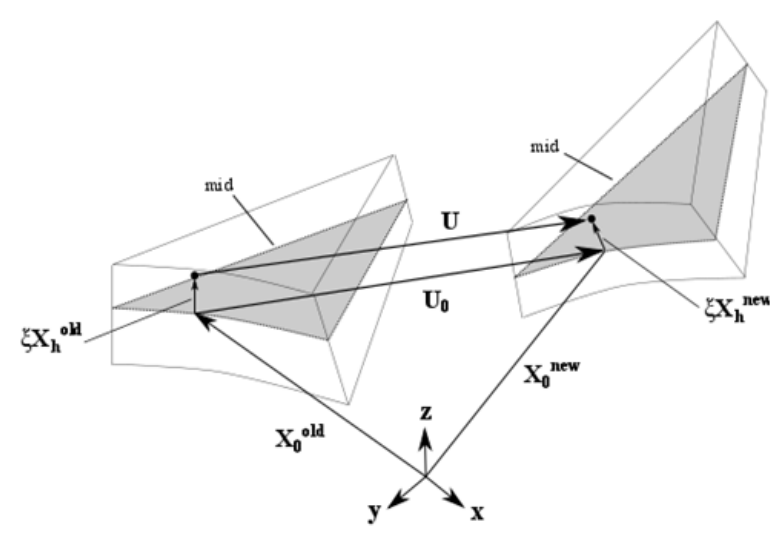

Fig. 1. Shell kinematics

$U=U_{0}+\xi U_{h}=\left(\begin{array}{c}u(x, y, z) \\ v(x, y, z) \\ w(x, y, z)\end{array}\right)=\left(\begin{array}{c}u_{0}(x, y, z) \\ v_{0}(x, y, z) \\ w_{0}(x, y, z)\end{array}\right)+\xi\left(\begin{array}{c}r_{y}(x, y) \\ -r_{x}(x, y) \\ 0\end{array}\right)$

where, $u_{0}, v_{0}, w_{0}$ are the mid-surface displacements, $r_{x}$, $r_{y}$ are the rotations of the shell mid-surface and $\xi$ the transverse direction vector which is normal to the midsurface. The linear strain field consisting of membrane strains $\varepsilon_{m}$, flexural strains $\xi \kappa_{b}$ and shear strains $\gamma$ can be expressed in the following form:

$\left(\begin{array}{c}\varepsilon_{x} \\ \varepsilon_{y} \\ \gamma_{x y}\end{array}\right)=\varepsilon=\varepsilon_{m}+\xi \kappa_{b}=\left(\begin{array}{c}u_{0, x} \\ v_{0, y} \\ u_{0, y}+v_{0, x}\end{array}\right)+\xi\left(\begin{array}{c}r_{y, x} \\ -r_{x, y} \\ r_{y, y}-r_{x, x}\end{array}\right)$

$\left(\begin{array}{l}\gamma_{y z} \\ \gamma_{x z}\end{array}\right)=\gamma=\left(\begin{array}{l}w_{0, y}+r_{y} \\ w_{0, x}-r_{x}\end{array}\right)$

For the laminated composite shell, the constitutive equation for each layer $(k)$ is obtained starting from the Hooke's law and implementing the plane stress assumption. It reads:

$\left[\begin{array}{c}\sigma_{x x} \\ \sigma_{y y} \\ \tau_{x y} \\ \tau_{y z} \\ \tau_{x z}\end{array}\right]_{(k)}=\left[\begin{array}{ccccc}Q_{11} & Q_{12} & Q_{16} & 0 & 0 \\ Q_{21} & Q_{22} & Q_{26} & 0 & 0 \\ Q_{16} & Q_{26} & Q_{66} & 0 & 0 \\ 0 & 0 & 0 & Q_{44} & Q_{45} \\ 0 & 0 & 0 & Q_{54} & Q_{55}\end{array}\right]_{(k)}\left[\begin{array}{c}\varepsilon_{x x} \\ \varepsilon_{y y} \\ \gamma_{x y} \\ \gamma_{y z} \\ \gamma_{x z}\end{array}\right]_{(k)}=\mathrm{Q}\left[\begin{array}{c}\varepsilon \\ \gamma\end{array}\right]$

where, the material constants are:

$$
\begin{gathered}
Q_{11}=\frac{E_{1}}{1-v_{21} v_{12}} \quad Q_{12}=\frac{E_{2} v_{12}}{1-v_{21} v_{12}} \quad Q_{22}=\frac{E_{2}}{1-v_{21} v_{12}} \\
Q_{66}=G_{12} \quad Q_{44}=G_{23} \quad Q_{55}=G_{13}
\end{gathered}
$$


and where $G_{12}, G_{23}, G_{13}$ are the shear moduli in the $x_{e}-y_{e}$, $y_{e^{-}} z_{e}, x_{e}-z_{e}$ planes (local reference coordinates), $E_{1}, E_{2}$ are the Young's moduli in the $x_{e^{-}}$and $y_{e^{-}}$-direction respectively and $v_{12}, v_{21}$ are the Poisson's ratios.

Laminates are commonly made of several orthotropic layers with different fiber orientation with respect to the reference element system $\left(x_{e}, y_{e}, z_{e}\right)$. The material matrix $Q^{\prime}$ of the $\mathrm{k}^{\text {th }}$ layer given with respect to the reference frame is simply the result of in-plane rotation:

$$
\begin{aligned}
& {\left[\begin{array}{c}
\sigma_{x x} \\
\sigma_{y y} \\
\tau_{x y} \\
\tau_{y z} \\
\tau_{x z}
\end{array}\right]_{(k)}=\left[\begin{array}{ccccc}
Q_{11}^{\prime} & Q_{12}{ }^{\prime} & Q_{16}{ }^{\prime} & 0 & 0 \\
Q_{21}{ }^{\prime} & Q_{22}{ }^{\prime} & Q_{26}{ }^{\prime} & 0 & 0 \\
Q_{16}{ }^{\prime} & Q_{26}{ }^{\prime} & Q_{66}{ }^{\prime} & 0 & 0 \\
0 & 0 & 0 & Q_{44^{\prime}} & Q_{45}{ }^{\prime} \\
0 & 0 & 0 & Q_{54}{ }^{\prime} & Q_{55}{ }^{\prime}
\end{array}\right]_{(k)}\left[\begin{array}{c}
\varepsilon_{x x} \\
\varepsilon_{y y} \\
\gamma_{x y} \\
\gamma_{y z} \\
\gamma_{x z}
\end{array}\right]_{(k)}} \\
& =\mathrm{Q}^{\prime}\left[\begin{array}{c}
\varepsilon \\
\gamma
\end{array}\right]=\mathrm{Q}^{\prime} \varepsilon^{*}
\end{aligned}
$$

\section{Piezoelectric Equations}

Laminates are commonly made of several orthotropic layers. However, this work considers laminates that involve piezoelectric layers as well. The linear piezoelectric constitutive equations can be expressed in the following form:

$\left[\begin{array}{c}\sigma \\ \mathrm{D}\end{array}\right]=\left[\begin{array}{cc}\mathrm{c} & -\mathrm{e}^{T} \\ \mathrm{e} & \mathrm{g}\end{array}\right]\left[\begin{array}{c}\varepsilon^{*} \\ \mathrm{E}\end{array}\right]$

where, $\sigma$ and $\varepsilon^{*}$ are the stress and strain vectors, respectively, $D$ and $E$ are the dielectric displacement and electric field vectors, respectively and $c, e, g$ are the elasticity matrix, the piezoelectric coupling coefficients matrix and the dielectric constants matrix, respectively. Mostly, the piezoelectric constant matrix $e$ is not given explicitly, but is expressed with piezoelectric strain constant matrix $d$ as follows:

$$
e=d Q_{i p}^{\prime}
$$

where, $d$ reads:

$$
d=\left[\begin{array}{ccc}
0 & 0 & 0 \\
0 & 0 & 0 \\
d_{31} & d_{32} & 0
\end{array}\right]
$$

\section{Electric Field}

A constant electric field across the thickness of the piezolayers is assumed, thus implying a linear distribution of the electric potential. The relation between the two is described in the following manner:

$$
E=-B_{\varphi} \varphi_{k}
$$

where, $B_{\varphi}$ is the electric field-electric potential matrix and $\varphi_{k}$ is the vector of electric potential differences between the top and bottom surfaces of the k-th piezolayer.

\section{Strain Energy}

The strain energy of an element, $U_{e}$, can be expressed as:

$$
\begin{aligned}
U_{e} & =\frac{1}{2} \int_{V} \varepsilon^{*^{T}} c \varepsilon^{*} d V-\int_{V p} \varepsilon^{*^{T}} e^{T} E d V \\
& =\frac{1}{2} u_{e}^{T} \int_{V} B^{T} c B d V u_{e}-u_{e}^{T} \int_{V p} B^{T} e^{T} B_{\varphi} d V \varphi_{e} \\
& =\frac{1}{2} u_{e}^{T} K_{u u, e} u_{e}-u_{e}^{T} K_{\text {upe }} \varphi_{e}
\end{aligned}
$$

where, $B$ is the mechanical strain-displacement matrix and $u_{e}$ and $\varphi_{e}$ are the nodal displacement vector and the potential per piezoelectric layer for an element, respectively, while $V$ and $V_{p}$ are the volume of the element and the volume of the piezoelectric layer in the element, respectively. $K_{u u, e}$ is the mechanical stiffness matrix and $K_{u \varphi, e}$ is the piezoelectric coupling stiffness matrix of an element.

\section{Electric Energy}

Based on the constitutive relations, straindisplacement and electric field-electric potential relations, the element electric energy per element $W_{e}$ can be expressed as:

$$
\begin{aligned}
W_{e} & =\int_{\mathrm{Vp}} \mathrm{E}^{T} \mathrm{e} \varepsilon * d V+\frac{1}{2} \int_{\mathrm{Vp}_{\mathrm{p}}} \mathrm{E}^{\mathrm{T}} \mathrm{gE} d V \\
& =\varphi_{\mathrm{e}}^{\mathrm{T}} \mathrm{K}_{\varphi \mathrm{u}, \mathrm{e}} \mathrm{u}_{e}-\frac{1}{2} \varphi_{e}^{T} \mathrm{~K}_{\varphi \varphi, \mathrm{e}} \varphi_{\mathrm{e}}
\end{aligned}
$$

\section{Work done by the External Forces and Electric Charge}

The virtual work done by external forces $F_{\text {ext,e }}$ and applied electric charge $Q_{\varphi, e}$ is given by:

$$
W_{e x t}=u_{e}^{T} F_{e x t e}+\varphi_{e}^{T} Q_{\varphi, e}
$$

The finite element equations for a static case involving a piezoelectric continuum are obtained using the variational approach. For the sake of brevity, only the resulting form is given here (Marinkovic et al., 2006):

$$
\begin{aligned}
& K_{u u, e} u_{e}+K_{u \varphi, e} \varphi_{e}=F_{e x t, e} \\
& K_{\varphi \varphi, e} u_{e}+K_{\varphi \varphi, e} \varphi_{e}=Q_{\varphi, e}
\end{aligned}
$$




\section{CS-DSG3 Element}

In the CS-DSG3 formulation, the element domain is divided into three DSG3 (Nguyen-Thoi et al., 2013) sub-triangle elements by connecting the triangle central point with the element nodes. The DSG formulation is applied in each of the sub-triangles. Afterwards, in order to smooth the strains in the sub-triangles, strain smoothing technique on the whole triangular element is applied. As a result, the CS-DSG3 overcomes the drawback of the DSG3 element that the results are dependent on the node numbering. A detailed description of the CS-DSG3 element formulation is given in (Rama et al., 2016). The CS-DSG3 element stiffness matrix is given as:

$$
\begin{aligned}
& \mathrm{K}_{\mathrm{e}, \mathrm{CS}-\mathrm{DSG} 3} \\
& =\mathrm{A}_{\mathrm{e}} \sum_{\mathrm{k}=1}^{\mathrm{n}} \mathrm{T}^{\mathrm{T}}\left(\begin{array}{l}
\mathrm{B}_{\mathrm{m}^{*}}^{\mathrm{T}} \mathrm{D}_{\mathrm{m}}^{(\mathrm{k})} \mathrm{B}_{\mathrm{m}^{*}}+\mathrm{B}_{\mathrm{m}^{*}}^{\mathrm{T}} \mathrm{B}^{(\mathrm{k})} \mathrm{B}_{\mathrm{b}^{*}} \\
+\mathrm{B}_{\mathrm{b}^{*}}^{\mathrm{T}} \mathrm{B}^{(\mathrm{k})} \mathrm{B}_{\mathrm{m}^{*}}+\mathrm{B}_{\mathrm{b}^{*}}^{\mathrm{T}} \mathrm{D}_{\mathrm{b}}^{(\mathrm{k})} \mathrm{B}_{\mathrm{b}^{*}}+\mathrm{B}_{\mathrm{s}^{*}}^{\mathrm{T}} \mathrm{D}_{\mathrm{s}}^{(\mathrm{k})} \mathrm{B}_{\mathrm{s}^{*}}
\end{array}\right) \mathrm{T}
\end{aligned}
$$

where, $T$ is the transformation matrix of coordinates from the global coordinate system $(x, y, z)$ to the local element coordinate system $\left(x_{e}, y_{e}, z_{e}\right)$ and $B_{m^{*}}, B_{b^{*}}, B_{s^{*}}$ are the membrane, bending and shear strain displacement matrices. The material matrices for the $k^{\text {th }}$ layer are obtained by integration of the elasticity matrix $c$ in the thickness direction:

$$
\begin{aligned}
& D_{m i j}^{(k)}=\int_{-h_{k} / 2}^{h_{k} / 2} Q_{i j}{ }^{\prime} d z=h_{k} Q_{i j}{ }^{\prime} \\
& B_{i j}^{(k)}=\int_{-h_{k} / 2}^{h_{k} / 2} z Q_{i j}{ }^{\prime} d z=\frac{z_{k+1}-z_{k}}{2} h_{k} Q_{i j} \\
& D_{b j}^{(k)}=\int_{-h_{k} / 2}^{h_{k} / 2} z^{2} Q_{i j}{ }^{\prime} d z \\
& D_{s j}^{(k)}=k \int_{-t / 2}^{t / 2} Q_{i j}{ }^{\prime} d z=k h_{k} Q_{i j}{ }^{\prime} \quad(i, j=1,2,6)
\end{aligned}
$$

The piezoelectric coupling is achieved between the in-plain strain field and electric field. As a result, the piezoelectric coupling and dielectric stiffness matrices $K_{u \varphi}, K_{\varphi \varphi}$ are obtained:

$$
\begin{aligned}
& K_{e, u \varphi}=\int_{V}\left[\begin{array}{ll}
B_{m^{*}} & B_{b^{*}}
\end{array}\right]^{T} e^{T} B_{\varphi} d V \\
& K_{e, \varphi \varphi}=\int_{V} B_{\varphi}{ }^{T} g B_{\varphi} d V
\end{aligned}
$$

where, a typical term of $B_{\varphi}$ for the $k^{\text {th }}$ layer is $\left(-1 / h_{k}\right)$

\section{Co-Rotational FEM Formulation}

In the CR FEM formulation, a local reference frame is attached to the material and follows the rigid-body motion of the material. In that manner it is possible to separate the rigid-body motion from the deformable motion. Provided the strains are small despite relative large displacements, the approach allows the use of engineering stress and strain measures. At the same time, the geometrical and materials nonlinearities are separated, thus facilitating later extension of the formulation into the realm of materially nonlinear behavior. The resolution of accounting for the rigid-body motion may differ. This is typically done by proving a local reference frame at each element integration point, but the present formulation the approach is simplified by providing a single local frame for each finite element. Its motion represents an average element rigid-body rotation. In this manner, the elastic behavior of each element remains linear with respect to the local element frame attached to the element and following its rigidbody motion. Given the rotational matrix, $R_{e}$, which describes the rigid-body rotation of an element, the element stiffness matrix is updated as follows:

$K_{e}^{R}=R_{e} K_{e} R_{e}^{T}$

Hence, the deformation of an element with respect to the local, co-rotational frame is described by a linear model. The rotational matrix, current and initial configuration, $x_{e}$ and $x_{0 e}$, are used to determine the rotation-free displacements with respect to the initial configuration as:

$u^{0}=R_{e}^{T} x_{e}-x_{0 e}$

Finally, the internal forces with respect to the initial configuration are computed using the linear stiffness matrix and rotation-free displacements and are furthermore rotated to obtain the internal forces with respect to the current configuration:

$F_{e}=R_{e} K_{e} u^{0}$

Update of the rotational degrees of freedom is more demanding compared to translations. The shell normals at each node are updated by means of incremental rotations computed in each time-step. The incremental rotational matrix of a shell normal is determined as follows (Argyris, 1982):

$Q=I+\frac{\sin \gamma}{\gamma} S+\frac{1}{2}\left(\frac{\sin (\gamma / 2)}{(\gamma / 2)}\right)^{2} S^{2}$

Where: 


$$
\gamma=\sqrt{r_{g, x}^{2}+r_{g, y}^{2}+r_{g, z}^{2}}
$$

and:

$$
S=\left[\begin{array}{ccc}
0 & -r_{g, z} & r_{y} \\
r_{z} & 0 & -r_{g, x} \\
-r_{g, y} & r_{g, x} & 0
\end{array}\right]
$$

with $r_{g, x}, r_{g, y}$ and $r_{g, z}$ denoting the 3 incremental global nodal rotations. The shell node normal is updated using the rotational matrix $Q$. Also, the initial normal at the node is rotated through the element rigid-body rotation. The soobtained direction is compared to the actual shell normal to get the deformational nodal rotations, free of rigidbody rotation. This enables the computation of internal nodal moments, which are needed to proceed with the geometrically nonlinear computation.

Once the global tangential stiffness matrix and the vector of internal forces are updated, geometrically nonlinear FEM computation may proceed. Thin-walled structures are known for their susceptibility to large rotations, whereby the strains remain small. This is why they represent a suitable candidate for application of the proposed formulation. The formulation has been implemented with the CS-DSG3 shell element.

\section{Numerical Examples}

Several examples have been studied to assess the performance of the CS-DSG3 in linear and co-rotational nonlinear static piezoelectric coupled analysis.

\section{Static Linear Cases}

With the given predefined electric voltage, $\varphi_{e}$, the element loads induced through the inverse piezoelectric effect (actuation) are computed in the following manner:

$$
F_{e, \varphi}=-K_{e, u \varphi} \varphi_{e}
$$

Afterwards the element loads are assembled to the global force vector.

\section{Piezoelectric Bimorph Beam}

The piezoelectric bimorph pointer consists of two uniaxial piezoelectric layers with opposite polarization. Such a structure can be used as a bending actuator or sensor. The geometry of the bimorph beam is presented in Fig. 2. The length $\mathrm{L}$ of the beam is $0.1 \mathrm{~m}$, the total height $\mathrm{H}$ is $0.1 \mathrm{~mm}$ and the width $\mathrm{W}$ is $5 \mathrm{~mm}$. The beam layers are made of uniaxial PVDF with opposite polarities. Table 3 presents the material properties of PVDF. The bimorph beam acts as an actuator, with a voltage of $1 \mathrm{~V}$ applied over its thickness.
The obtained results are given in Table 1 and also depicted in Fig. 3. Using Bernoulli-Euler beam theory and the constitutive equations of linear piezoelectricity, the analytical solution for the deflection w along the length is obtained:

$w(x)=-\frac{3}{2} \frac{e_{31} \varphi}{h^{2}} x^{2}$

The expression yields the absolute tip deflection $\left|w\left(x_{\text {end }}\right)\right|$ of $3.45 \mathrm{E}-7 \mathrm{~m}$. The result by the presented shell element coincides with the analytical solution.

\section{Simple Supported Piezoelectric Composite Plate (sym)}

A simply supported square laminated piezoelectric plate (dimensions $a \times a=0.2 \times 0.2 \mathrm{~m}$ ), subjected to a uniform load $q_{D}=100 \mathrm{~N} / \mathrm{m}^{2}$, is considered. The plate has symmetrically bonded piezoelectric ceramics on the outer surfaces and four composite layers in addition to two piezolayers. The stacking sequence of the composite plate is $\left[\mathrm{p} /-45^{\circ} / 45^{\circ}\right]_{\mathrm{sym}}$ (Fig. 4). The layers denoted by $\mathrm{p}$ are the piezoelectric layers and the subscript 'sym' indicates a symmetric sequence of layers. The total thickness of the plate is $1.2 \mathrm{~mm}$. Each composite (passive) layer has the same thickness of $0.25 \mathrm{~mm}$ and is made of T300/976 graphite/epoxy. The piezoelectric layers have a thickness of $0.1 \mathrm{~mm}$ and are made of PZTG1195N (material properties in Table 3). Figure 5 displays the central line deflection of the considered structure subjected to the combined excitation involving the uniform load and different input voltages of 0,5 and $10 \mathrm{~V}$. The obtained results agree well with those obtained by (Phung-Van et al., 2015) and (Liu et al., 2004) (Table 1).

\section{Clamped Piezoelectric Composite Plate (Asymmetric)}

A uniform load $q_{D}=100 \mathrm{~N} / \mathrm{m}^{2}$ acts upon a cantilevered square laminate piezoelectric plate $(a \times a=$ $0.2 \times 0.2 \mathrm{~m}$ ). The plate has symmetrically bonded piezoelectric ceramics on the outer surfaces and additional four composite layers. The stacking sequence of the composite plate is in this case $\left[\mathrm{p} /-45^{\circ} / 45^{\circ}\right]_{\text {as }}$ (Fig. $6)$. The subscript 'as' stands here for an antisymmetric laminate. The total thickness of the plate is $1.2 \mathrm{~mm}$. The passive layers have the same thickness of $0.25 \mathrm{~mm}$ and are made of T300/976 graphite/epoxy. The piezoelectric composite layers have a thickness of 0.1 $\mathrm{mm}$ and are made of PZTG1195N. Figure 7 depicts the central line deflection of the plate additionally subjected to the input voltages of 0,30 and $50 \mathrm{~V}$. The tip point deflection is presented in Table 1 . The obtained results are in good agreement with those obtained by (Lam et al., 1997; Liu et al., 2004) (Table 1). 


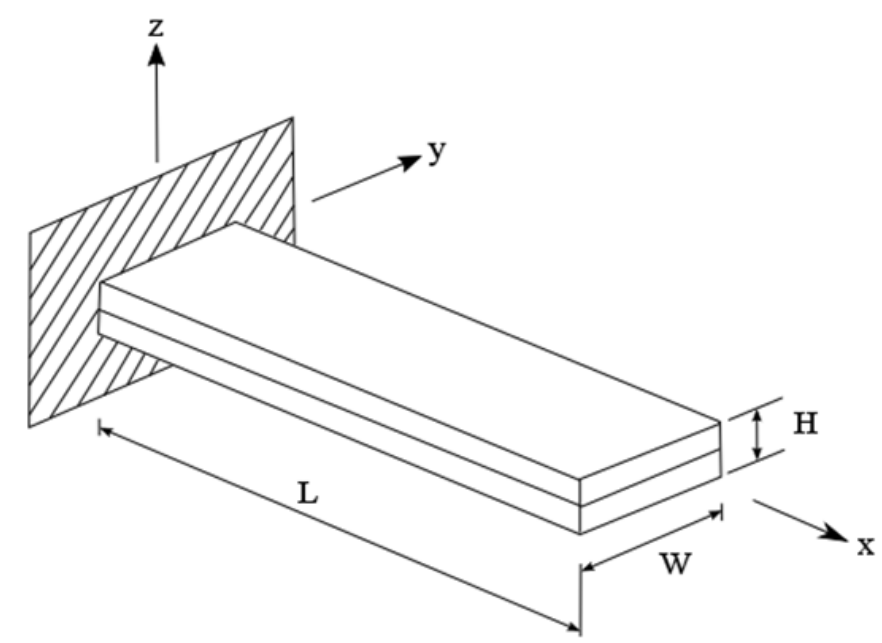

Fig. 2. Geometry of the piezoelectric bimorph beam

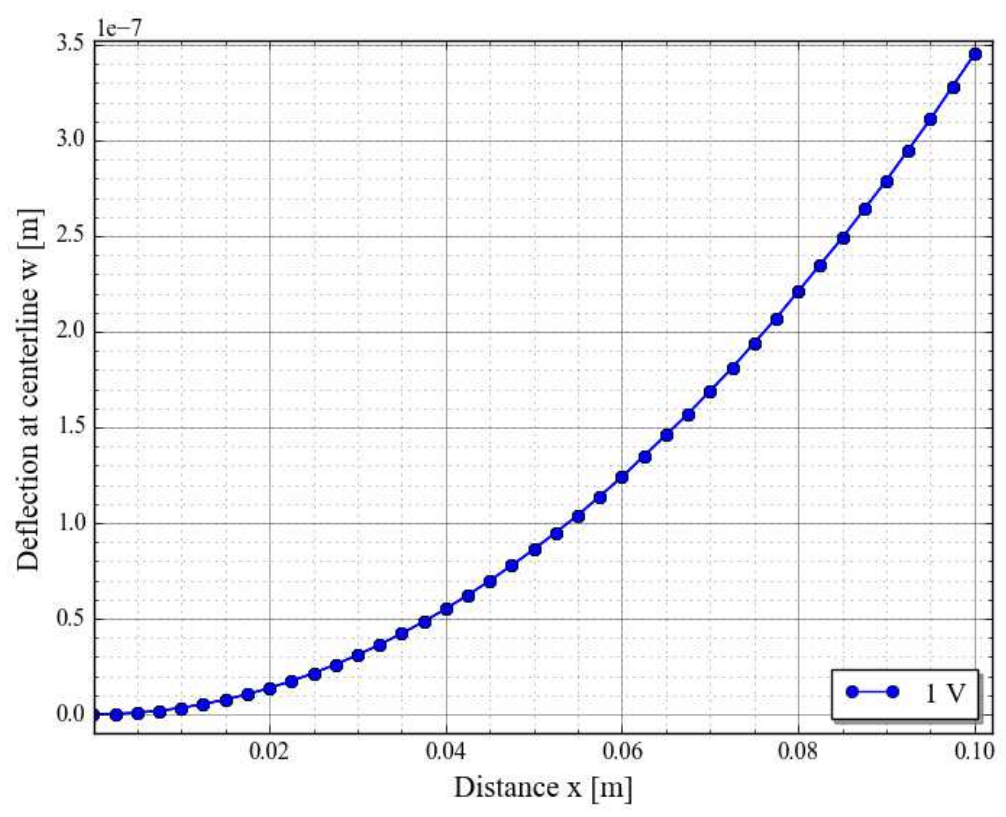

Fig. 3. Centerline deflection of bimorph beam subjected to input voltage of $1 \mathrm{~V}$

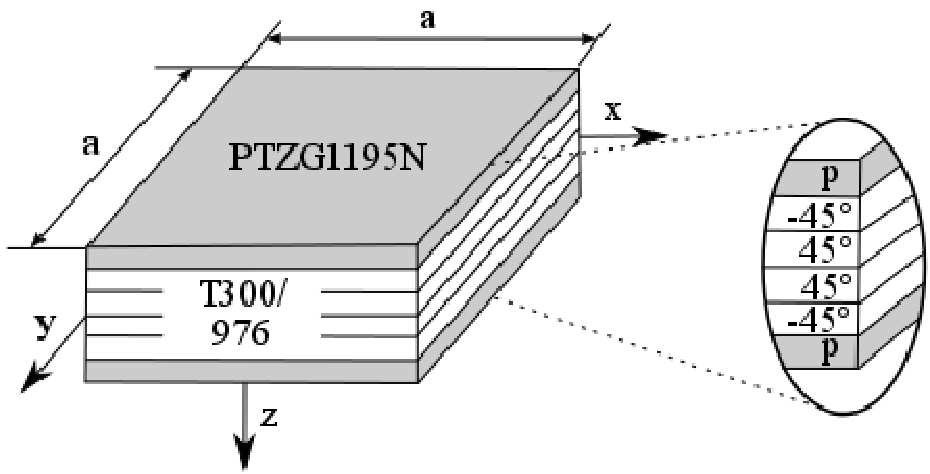

Fig. 4. Sequence of layers of the simply supported piezoelectric composite plate 


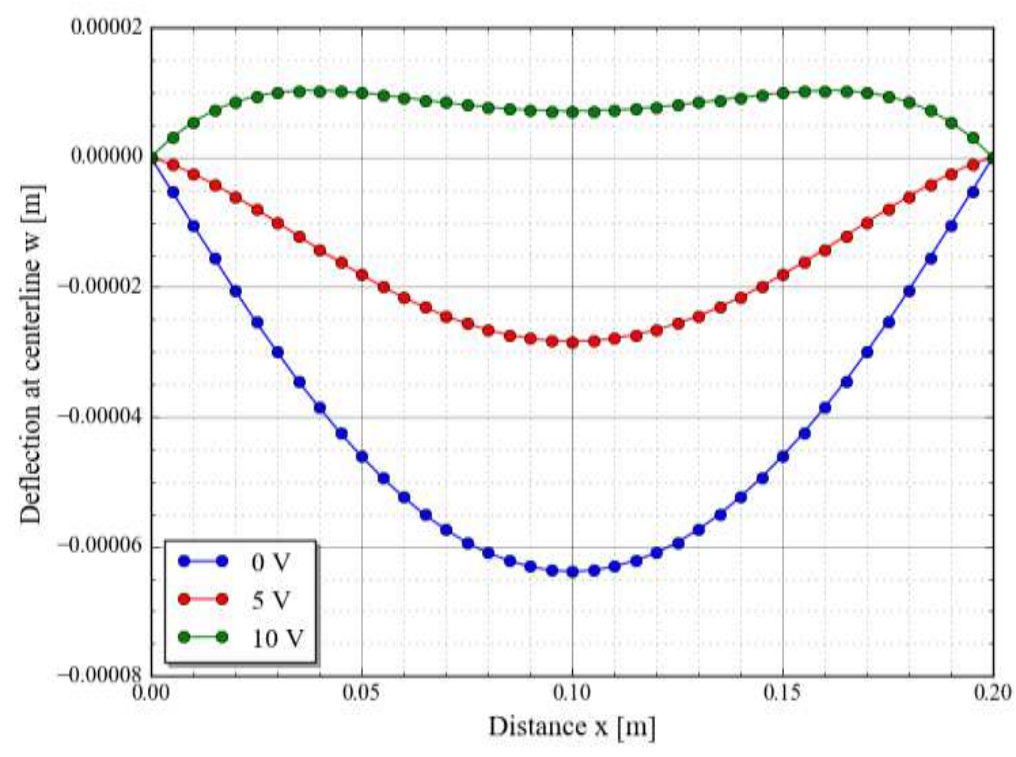

Fig. 5. Centerline deflection of simply supported laminated plate subjected to a uniform load and different input voltages

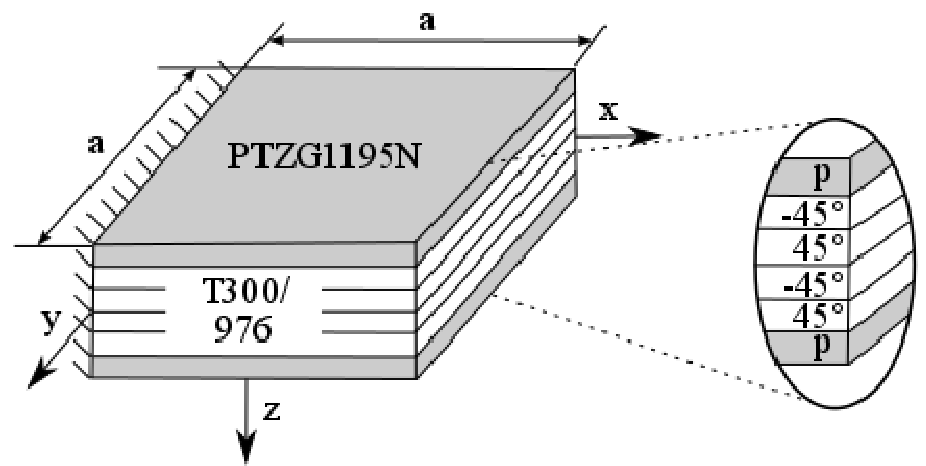

Fig. 6. Antisymmetric sequence of layers of the clamped piezoelectric composite plate

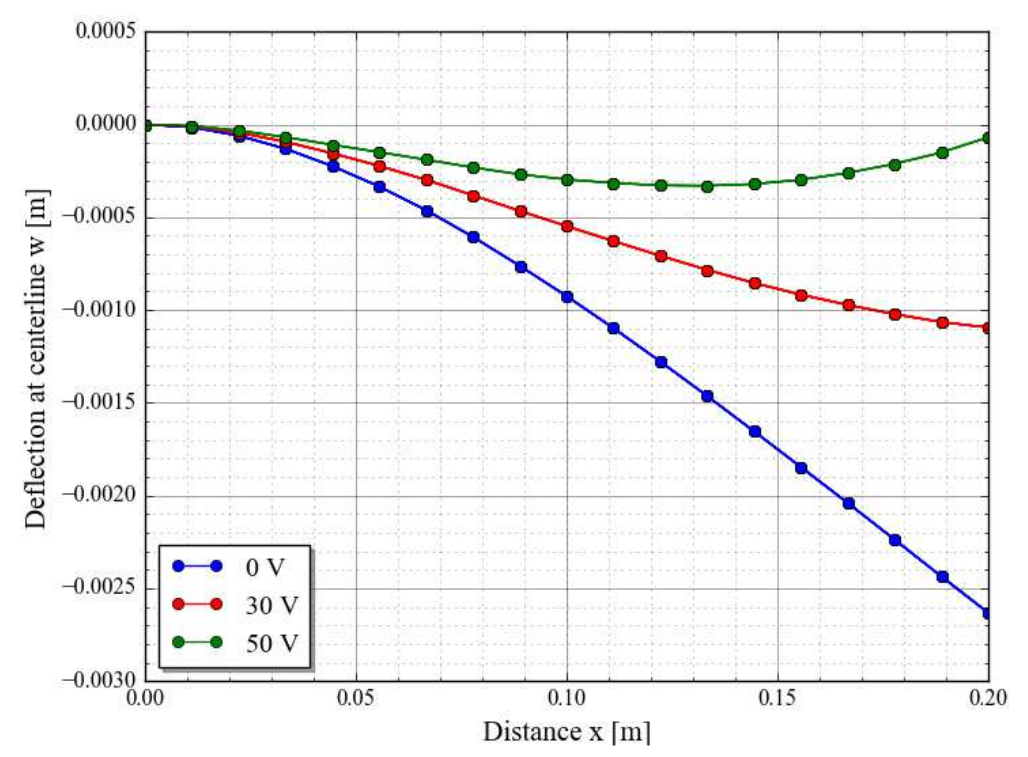

Fig. 7. Centerline deflection of clamped plate subjected to a uniform load and different input voltages 
Table 1. Static linear case-results and reference solutions

\begin{tabular}{llll}
\hline Bimorph Beam & Present & Analytical & \\
\hline Tip node deflection w [m] & $3.45 \mathrm{E}-7$ & $3.45 \mathrm{E}-7$ & \\
Simple supported composite plate & Present & Phung-Van et al. $(2015)$ & Liu et al. $(2004)$ \\
Central node deflection w [m] & & & $-0.604 \mathrm{E}-4$ \\
$0 \mathrm{~V}$ & $-0.639 \mathrm{E}-04$ & $-0.633 \mathrm{E}-4$ & $-0.272 \mathrm{E}-4$ \\
$5 \mathrm{~V}$ & $-0.284 \mathrm{E}-04$ & $-0.280 \mathrm{E}-4$ & $-0.757 \mathrm{E}-5$ \\
$10 \mathrm{~V}$ & $-0.714 \mathrm{E}-05$ & $-0.721 \mathrm{E}-5$ & Liu et al. $(2004)$ \\
Clamped composite plate & Present & Lam et al. $(1997)$ & $-0.271 \mathrm{E}-4$ \\
Central node deflection w [m] & & & $-0.105 \mathrm{E}-4$ \\
$0 \mathrm{~V}$ & $-0.263 \mathrm{E}-04$ & $-0.278 \mathrm{E}-4$ & $-0.440 \mathrm{E}-6$ \\
$30 \mathrm{~V}$ & $-0.109 \mathrm{E}-04$ & $-0.110 \mathrm{E}-4$ & $-0.510 \mathrm{E}-6$ \\
$50 \mathrm{~V}$ & $-0.690 \mathrm{E}-06$ & &
\end{tabular}

Table 2. Static nonlinear cases-Results and reference solutions

\begin{tabular}{lll}
\hline Simple supported (2 sides) composite plate & Present & (Marinkovic et al., 2008) \\
\hline $\begin{array}{l}\text { Central node deflection w [m] 300 V } \\
\text { Clamped composite plate }\end{array}$ & $3.705 \mathrm{E}-04$ & $3.700 \mathrm{E}-04$ \\
Tip node deflection w [m] & Present & \\
$300 \mathrm{~V}$ & $0.271 \mathrm{E}-2$ & $0.270 \mathrm{E}-2$ \\
$\begin{array}{l}\text { Tip node deflection v }[\mathrm{m}] \\
300 \mathrm{~V}\end{array}$ & $0.621 \mathrm{E}-5$ & $0.650 \mathrm{E}-5$ \\
$\begin{array}{l}\text { Tip node deflection } \mathrm{u}[\mathrm{m}] \\
300 \mathrm{~V}\end{array}$ & $0.121 \mathrm{E}-3$ & $0.120 \mathrm{E}-5$ \\
\hline
\end{tabular}

Table 3. Material properties of piezoelectric and composite materials

\begin{tabular}{|c|c|c|c|}
\hline Properties & PVDF & PTZ-G1195 & T300/976 \\
\hline \multicolumn{4}{|l|}{ Young's moduli [GPa] } \\
\hline $\mathrm{E}_{\mathrm{xx}}$ & 2.0 & 63.0 & 150.0 \\
\hline $\begin{array}{l}\mathrm{E}_{\mathrm{yy}}=\mathrm{E}_{\mathrm{zz}} \\
\text { Poisson's ratio [-] }\end{array}$ & 2.0 & 63.0 & 9.0 \\
\hline $\begin{array}{l}v_{x y}=v_{x z}=v_{y z} \\
\text { Shear moduli }[\mathrm{GPa}]\end{array}$ & 0.29 & 0.3 & 0.3 \\
\hline $\mathrm{G}_{\mathrm{xy}}=\mathrm{G}_{\mathrm{xz}}$ & 1.0 & 24.2 & 7.1 \\
\hline $\begin{array}{l}\mathrm{G}_{\mathrm{yz}} \\
\text { Piezoelectric coefficients }\end{array}$ & 1.0 & 24.2 & 2.5 \\
\hline $\begin{array}{l}\mathrm{d}_{31}=\mathrm{d}_{32}[\mathrm{~m} / \mathrm{V}] \\
\text { Electric permittivities }\end{array}$ & 0.046 & $2.54 \mathrm{E}-10$ & \\
\hline $\begin{array}{l}\mathrm{p}_{11}=\mathrm{p}_{22}=\mathrm{p}_{33}[\mathrm{~F} / \mathrm{m}] \\
\text { Piezoelectric constants }\end{array}$ & $0.1062 \mathrm{E}-9$ & $15.3 \mathrm{E}-9$ & \\
\hline $\mathrm{e}_{31}=\mathrm{e}_{32}\left[\mathrm{C} / \mathrm{m}^{2}\right]$ & 0.046 & 22.86 & \\
\hline
\end{tabular}

\section{Nonlinear Static Cases}

The piezoelectrically induced loads $F_{\varphi}$ are of the follower type (configuration dependent). This requires caution in the choice of the increment size in the analysis. In each load step the piezoelectrically induced element loads are calculated, with the given electric voltage $\varphi$, as following:

$$
F_{e,}=-R_{e} K_{e, u \varphi} \varphi_{e}
$$

Afterwards the element loads are assembled to the global force vector.

\section{Simple Supported (at two Edges) Piezoelectric Composite Plate}

Laminated piezoelectric square plate is simply supported along two opposite edges parallel to the $\mathrm{x}$ - axis. The edge length is $a=0.4 \mathrm{~m}$. It is subjected to an input voltage of $300 \mathrm{~V}$. The structure is made of three layers, the outer two are made of PZT-G1195N and have a thickness of $0.2 \mathrm{~mm}$ while the mid-layer is made of T300/976 graphite-epoxy and has a thickness of 0.15 $\mathrm{mm}$ and fiber orientation of $90^{\circ}$, with the $\mathrm{x}$-axis taken as the reference direction. The load-displacement $(|w|)$ curve for the representative point B (plate center point; Fig. 8) is presented in Fig. 9. The linear analysis yields the absolute value of the representative point $\mathrm{B}$ vertical displacement equals to $6.994 \mathrm{E}-04 \mathrm{~m}$ while the nonlinear prediction gives the result of $3.705 \mathrm{E}-04 \mathrm{~m}$. Marinkovic et al. (2008) this case is solved using the updated Lagrangian formulation. Compared to these result (Table 2), the difference is less than $0.1 \%$ in both linear and nonlinear static analyses. 


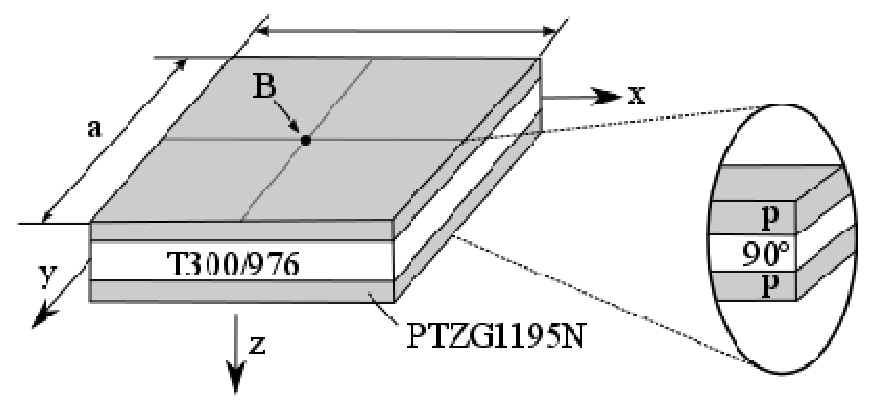

Fig. 8. Sequence of layers of square piezoelectric composite plate and representative point B

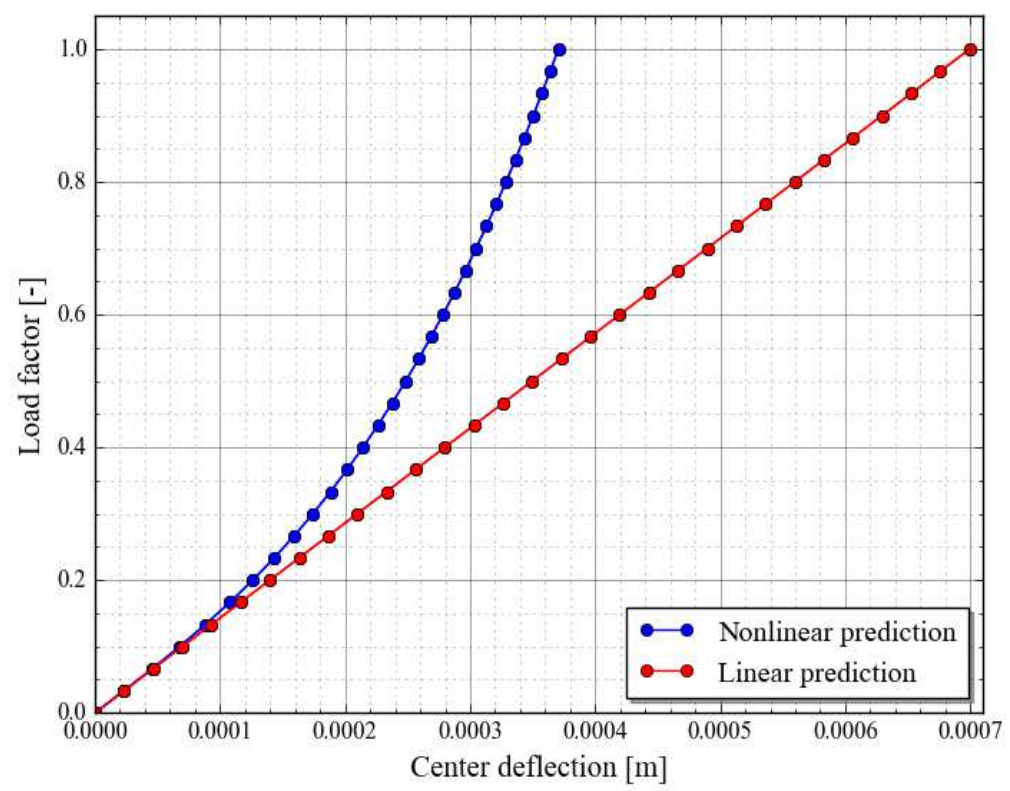

Fig 9. Linear and nonlinear answer of center node deflection simply supported structure subjected a by an input voltage of $300 \mathrm{~V}$

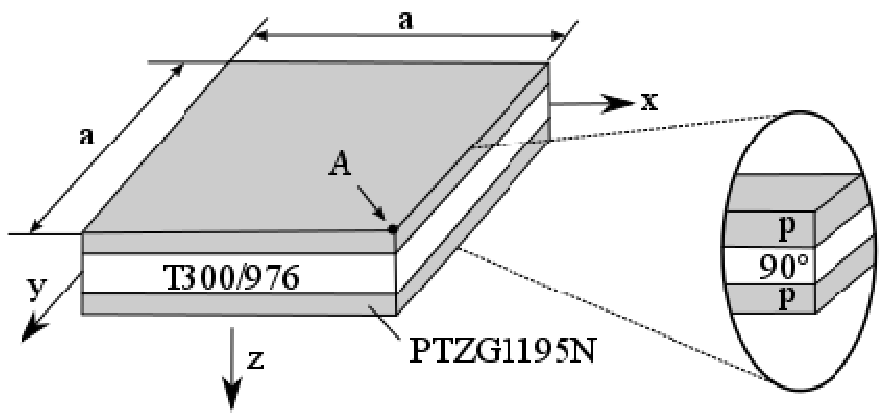

Fig. 10. Laminate configuration of square piezoelectric composite plate (clamped)-and position of representative point A

\section{Clamped Piezoelectric Composite Plate}

A cantilevered laminate piezoelectric plate of dimensions $a \times a=0.4 \times 0.4 \mathrm{~m}$ subjected to an input voltage of $300 \mathrm{~V}$ is considered. The plate is made of the same laminate as in the previously studied case. The load-displacement $(|w|)$ curve for the representative point A (Fig. 10) is presented in Fig. 11. The linear results for the representative point $\mathrm{A}$ vertical displacement yields $2.82 \mathrm{~mm}$ while the nonlinear prediction is $2.71 \mathrm{~mm}$. Compared to the results presented by (Marinković et al., 2008) the difference is less than $0.4 \%$ in the nonlinear and less than $0.8 \%$ in the linear case. 


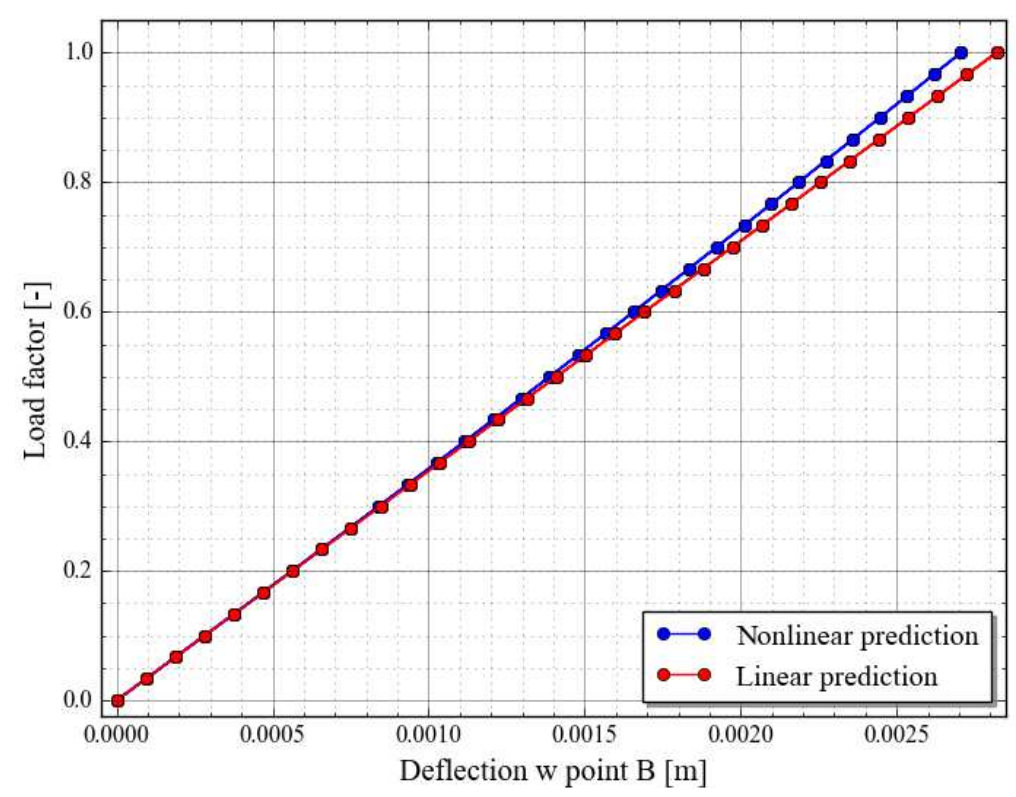

Fig. 11. Linear and nonlinear deflection of point $\mathrm{B}$ for the clamped structure subjected to input voltage of $300 \mathrm{~V}$

Figure 8 depicts the geometrically nonlinear results for the history of motion of the point $\mathrm{A}$ in the $\mathrm{x}$ - and $\mathrm{y}$-direction.

\section{Conclusion}

Design of smart structures requires efficient and reliable tools in order to analyze their behavior and assess various control algorithms. This paper offers such a modeling tool by extending an already available triangular shell type finite element. The extension allows modeling of piezoelectric laminated structures involving fiber-reinforced composite layers. Regarding piezolayers, the element covers patches polarized in the thickness direction and the assumption of constant difference of electric potentials between the upper and lower surface of a piezoelectric patch is used. It was demonstrated that the element provides highly accurate results for both linear and geometrically nonlinear results. Geometric nonlinearities are treated by means of CR-formulation, which is characterized by high efficiency and numerical stability.

The focus of this paper was put on static cases while further work will cover dynamic analysis as dynamics is where the advantages of smart structures particularly come to the fore. Further development should also cover piezolayers with the in-plane polarization. This would allow modeling of composite materials that involve piezoelectric fibers.

\section{Funding Information}

The authors have no support or funding to report.

\section{Authors' Contribution}

The first author of this manuscript has coded the applied shell type finite element. All authors made considerable contributions in integration of the developed element into an existing solver, choice of the verification examples and writing of the manuscript.

\section{Ethics}

The authors would like to disclose that Dr. Dragan Marinkovic (the second author) is a member of the editorial board for the American Journal of Engineering and Applied Sciences.

\section{References}

Allik, H. and T.J.R. Hughes, 1970. Finite element method for piezoelectric vibration. Int. J. Numerical Methods Eng., 2: 151-157. DOI: 10.1002/nme.1620020202

Argyris, J.H., 1982. An excursion into large rotations. Comput. Meth. Applied Mechan. Eng., 32: 85-155. DOI: 10.1016/0045-7825(82)90069-X

Benjeddou, A., 2000. Advances in piezoelectric finite element modeling of adaptive structural elements: A survey. Comput. Struct., 76: 347-363. DOI: $10.1016 / \mathrm{S} 0045-7949(99) 00151-0$

Gabbert, U., H. Köppe, F. Seeger and H. Berger, 2002. Modeling of smart composite shell structures. J. Theoretical Applied Mechan., 3: 575-593.

Kim, J.H. and Y.H. Kim, 2002. Three-node macro triangular shell element based on the assumed natural strains. Comput. Mechan., 29: 441-458. DOI: $10.1007 / \mathrm{s} 00466-002-0354-7$ 
Kogl, M. and M.L. Bucalem, 2003. Locking-free piezoelectric MITC shell elements. Proceedings of the 2nd MIT Conference on Computational Fluid and Solid Mechanics, Jun. 17-20, Amsterdam, Holand, pp: 392-395.

DOI: 10.1016/B978-008044046-0.50096-8

Kulkarni, S.A. and K.M. Bajoria, 2007. Large deformation analysis of piezolaminated smart structures using higher-order shear deformation theory. Smart Mater. Struct., 16: 1506-1516. DOI: 10.1088/0964-1726/16/5/002

Lam, K.Y., X.Q. Peng, G.R. Liu and J.N. Reddy, 1997. A finite-element model for piezoelectric composite laminates. Smart Mater. Struct., 6: 583-591. DOI: 10.1088/0964-1726/6/5/009

Liu, G.R., K.Y. Dal and K.M. Lim, 2004. Static and vibration control of composite laminates integrated with piezoelectric sensors and actuators using the radial point interpolation method. Smart Mater. Struct., 13: 1438-1447. DOI: 10.1088/0964-1726/13/6/015

Marinkovic, D., M. Zehn and Z. Marinkovic, 2012. Finite element formulations for effective computations of geometrically nonlinear deformations. Adv. Eng. Software, 50: 3-11.

DOI: $10.1016 /$ j.advengsoft.2012.04.005

Marinkovic, D. and Z. Marinkovic, 2012. On FEM modeling of piezoelectric actuators and sensors for thin-walled structures. Smart Struct. Syst., 9: 411-426. DOI: $10.12989 /$ sss.2012.9.5.411

Marinkovic, D., H. Köppe and U. Gabbert, 2006. Numerically efficient finite element formulation for modeling active composite laminates. Mechan. Adv. Mater. Struct., 13: 379-392.

DOI: $10.1080 / 15376490600777624$

Marinkovic, D., H. Köppe and U. Gabbert, 2007. Accurate modeling of the electric field within piezoelectric layers for active composite structures. J. Intelli. Mater. Syst. Struct., 18: 503-513.

DOI: $10.1177 / 1045389 X 06067139$
Marinkovic, D., H. Köppe and U. Gabbert, 2008. Degenerated shell element for geometrically nonlinear analysis of thin-walled piezoelectric active structures. Smart Mater. Struct., 17: 1-10. DOI: 10.1088/0964-1726/17/01/015030

Mesecke-Rischmann, S., 2004. Modellierung von flachen piezoelektrischen Schalen mit zuverlässigen finiten Elementen. PhD Thesis, Helmut-SchmidtUniversity in Hamburg.

Nestorovic, T., D. Marinković, S. Shabadi and M. Trajkov, 2014. User defined finite element for modeling and analysis of active piezoelectric shell structures. Meccanica, 49: 1763-1774. DOI: $10.1007 / \mathrm{s} 11012-014-9925-\mathrm{x}$

Nguyen-Thoi, T., P. Phung-Van, C. Thai-Hoang and H. Nguyen-Xuan, 2013. A Cell-based Smoothed Discrete Shear Gap method (CS-DSG3) using triangular elements for static and free vibration analyses of shell structures. Int. J. Mech. Sci., 74: 32-45. DOI: 10.1016/j.ijmecsci.2013.04.005

Nutti, B. and D. Marinković, 2014. An approach to efficient FEM simulations on graphics processing units using CUDA. Facta Universitatis Mechan. Eng., 12: 15-25.

Phung-Van, P., F.L. De Lorenzis, C.H. Thai, M. AbdelWahab and H. Nguyen-Xuan, 2015. Analysis of laminated composite plates integrated with piezoelectric sensors and actuators using higherorder shear deformation theory and isogeometric finite elements. Comput. Mater. Sci., 96: 495-505.

Piefort, V., 2001. Finite element modeling of piezoelectric active structures. PhD Thesis, Université Libre de Bruxelles.

Rabinovitch, O., 2005. Geometrically nonlinear behavior of piezoelectric laminated plates. Smart Mater. Struct., 14: 785-798.

Rama, G., D. Marinkovic and M. Zehn, 2016. Efficent corotational 3-node shell element. Am. J. Eng. Applied Sci., 9: 420-431. DOI: 10.3844/ajeassp.2016.420.431 